\title{
CICLO DE LOS NEGOCIOS EN COLOMBIA: EL PAPEL DE LA POLÍTICA DE ESTABILIZACIÓN*
}

\author{
Recibido: 21 de noviembre de 2014 • Aprobado: 10 de noviembre de 2015 \\ Jorge Mario Uribe ${ }^{* *}$ \\ Karol Carbonell ${ }^{* * *}$
}

\section{RESUMEN}

Este documento mide la sincronización entre las políticas de estabilización y el ciclo de los negocios en Colombia en el periodo comprendido entre marzo de 1990 y junio de 2013. Para la construcción de los ciclos se utiliza la metodología clásica y se construye un ciclo de referencia a partir de ciclos individuales de tres índices de actividad económica real. Con el fin de medir la sincronización entre el ciclo de negocios y los ciclos de las políticas fiscal y monetaria se utiliza el estadístico de Harding y Pagan (2006). Se concluye que durante el periodo de estudio las políticas de estabilización tienden a ser procíclicas (por el lado fiscal) y acíclicas (por el lado monetario). En la literatura se conoce a este fenómeno como when it rains, it pours y es común que se presente en economías emergentes como la colombiana.

\section{PALABRAS CLAVE}

Ciclo de los negocios Colombia, agregación de ciclos, política fiscal, política monetaria, economías emergentes.

\section{CLASIFICACIÓN JEL}

E23, E32, E52, E62, E63

\section{CONTENIDO}

Introducción; 1. Marco conceptual y revisión de la literatura; 2. Metodología; 3. Resultados; 4. Conclusiones; Bibliografía.

* Este artículo es producto del proyecto de investigación "Ciclos Financieros en América Latina" del grupo de investigación en Macroeconomía Aplicada y Economía Financiera (MAEF), clasificado en categoría B por Colciencias, de la Facultad de Ciencias Sociales y Económicas de la Universidad del Valle, Cali, Colombia.

** Economista, Universidad del Valle, Colombia. Ms en Economía, Universidad de los Andes, Colombia. M.Res. en Economía, Instituto Universitario Europeo, Italia. Profesor Asociado, Departamento de Economía, Universidad del Valle. Director del grupo de investigación MAEF. Correo electrónico: jorge.uribe@correounivalle.edu.co.

*** Economista, Universidad del Valle, Cali, Colombia. Monitora de Investigación en el grupo de MAEF. Correo electrónico: karol.carbonell@correounivalle.edu.co. 


\section{THE BUSINESS CYCLE IN COLOMBIA: THE ROLE OF THE STABILIZATION POLICY ABSTRACT}

This document measures the synchronization between the stabilization policy and the Colombian business cycle during the period March 1990 - June 2013. A classical methodology is used for the cycle construction and a reference cycle is constructed parting from three individual cycles of three real economic activity indicators. With the end of measuring the synchronization between the business cycle and the fiscal and monetary policy, the Harding and Pagan statistical analysis (2006) is used. It is concluded that for the period of time studied the stabilization policies tend to be pro-cyclic (for the fiscal case) and counter-cyclic (for the monetary case). This phenomenon is known in literature as when it rains, it pours and it is common to see in economies such as the Colombian.

\section{KEY WORDS}

Colombian business cycle, cycle aggregation, fiscal policy, monetary policy, emerging markets.

\section{JEL CLASSIFICATION}

E23, E32, E52, E62, E63

\section{CONTENT}

Introduction; 1. Conceptual framework and literature review; 2. Methodology; 3. Results; 4. Conclusions; Bibliography.

\section{CICLO DOS NEGÓCIOS NA COLÔMBIA: O PAPEL DA POLÍTICA DE ESTABILIZAÇÃO RESUMO}

Este documento mede a sincronização entre as políticas de estabilização e o ciclo dos negócios na Colômbia no período compreendido entre março de 1990 e junho de 2013. Para a construção dos ciclos se utiliza a metodologia clássica e se constrói um ciclo de referência a partir de ciclos individuais de três índices de atividade económica real. Com o fim de medir a sincronização entre o ciclo de negócios e os ciclos das políticas fiscais e monetárias se utiliza o estatístico de Harding e Pagan (2006). Se conclui que durante o período de estudo as políticas de estabilização tendem a ser pro-cíclicas (pelo lado fiscal) e contra-cíclicas (pelo lado monetário). Na literatura se conhece a este fenómeno como when it rains, it pours e é comum que se apresente em economias emergentes como a colombiana.

\section{PALAVRAS CHAVE}

Ciclo dos negócios Colômbia, agregaçã̃o de ciclos, política fiscal, política monetária, economias emergentes.

\section{CLASSIFICAÇÃO JEL}

E23, E32, E52, E62, E63

\section{CONTEÚDO}

Introdução; 1. Marco conceitual e revisão da literatura; 2. Metodologia; 3. Resultados; 4. Conclusões; Bibliografia. 


\section{INTRODUCCIÓN}

El estudio de los ciclos de los negocios trata sobre el comportamiento fluctuante de la actividad económica, así como las posibles consecuencias para la sociedad de tales fluctuaciones. Las teorías que lo componen hacen parte de los programas estándar en economía alrededor del mundo y constituyen las herramientas técnicas básicas para informar las decisiones de política, disponibles para banqueros centrales, reguladores y administradores de recursos fiscales. Los ciclos económicos se relacionan directamente con la capacidad de los agentes en una economía de mercado, para suavizar sus patrones intertemporales de consumo, y programar una senda óptima de inversión financiera y real. Se asocian también, de forma precisa, con las dinámicas del empleo, los precios, las tasas de interés y los salarios reales en las economías de mercado (Woodford, 2003; Galí, 2015). Por tales razones, el estudio de los ciclos es de gran interés para los economistas, en general, y para quienes diseñan y ejecutan las políticas económicas, en particular. De tal estudio se puede obtener información que contribuya con el diseño de herramientas que sirvan para estabilizar la actividad económica real y contribuyan así a generar un mayor nivel de bienestar para la economía en su conjunto.

No obstante, Colombia cuenta con relativamente pocas investigaciones que resuelvan cuestiones como: ¿se relacionan las políticas fiscal y monetaria con la dinámica de los ciclos económicos? o, ¿cuál variable de política tiene mayor efecto sobre los ciclos económicos? Este documento se propone avanzar en tal dirección. Se enfoca en descubrir si las medidas adoptadas, en la historia económica colombiana reciente, han sido eficaces, es decir, si han contribuido con la estabilización de la economía. Se busca, por tanto, responder interrogantes como: ¿̇uál es el grado de sincronización de las variables de política económica y el ciclo de los negocios colombiano? ¿son dichas variables de política procíclicas, contracíclicas o acíclicas?

Para cumplir con el objetivo planteado, es necesario tener en cuenta que no existe una herramienta única que permita fechar los ciclos. Así, se tiene que, dependiendo de la metodología utilizada, las fechas de inicio y finalización de las fases económicas, pueden variar. Un aporte importante de este documento es la aplicación de una metodología que permite utilizar series trimestrales para la creación de un ciclo de referencia económico único, sin necesidad de recurrir a técnicas estadísticas basadas en filtros. Se trata de la metodología clásica para fechar los ciclos, sustentada en un sencillo algoritmo de inspección visual de las series. En este sentido, es el único en la literatura colombiana, que partiendo de la metodología clásica, a nivel univariado, construye un ciclo de referencia más acorde con lo que se entiende como tal, a la hora de decretar una recesión. 
Este artículo se encuentra dividido en cuatro secciones sin contar esta introducción. En la primera se presenta el marco conceptual y la revisión de la literatura relacionada, la cual subraya el papel de las políticas de estabilización en la atenuación de los ciclos; en la segunda se expone la metodología utilizada para la construcción del ciclo de los negocios, y de las políticas fiscal y monetaria; en la tercera se presentan los resultados y en la cuarta las conclusiones.

\section{MARCO CONCEPTUAL Y REVISIÓN DE LA LITERATURA}

A principios del siglo XX, varios economistas como Spiethoff (1925) y Schumpeter (1935) intentaron proveer una definición precisa del ciclo económico. No obstante, fueron Burns y Mitchell (1946, p. 3) quienes denominaron al ciclo económico como "ciclo de negocios" y es quizá esta la definición más reconocida en la actualidad:

Los ciclos de negocios son un tipo de fluctuaciones que se presentan en la actividad económica agregada de las economías cuyo sistema productivo descansa principalmente en la empresas: un ciclo consta de expansiones que ocurren aproximadamente al mismo tiempo en muchas ramas de la actividad económica, seguidas de recesiones, contracciones y recuperaciones, también de carácter general, que conducen a la fase de expansión del siguiente ciclo; esta sucesión de fases es recurrente pero no periódica; la duración de los ciclos varía desde algo más de un año hasta diez o doce; no son divisibles en ciclos más cortos de carácter similar y con amplitudes aproximadamente iguales.

A la definición anterior se le conoce como clásica, en la literatura del ciclo económico. Además de esta definición se cuenta con conceptos básicos asociados con los ciclos, como lo son: auge, recesión, depresión y expansión. De esta forma se tiene que las fases ascendentes y descendentes del ciclo de los negocios, se pueden clasificar en cuatro:

- Auge o pico: es el momento más elevado (máximo) del ciclo económico.

- Recesión o desaceleración: es la fase descendente del ciclo. Cuando se presenta un crecimiento negativo, se le denomina recesión, pero si se trata de una disminución en la tasa de crecimiento, se le denomina desaceleración.

- Depresión o valle: es el punto más bajo (mínimo) del ciclo económico.

- Recuperación o expansión: es la fase ascendente del ciclo. Mientras se presente un signo negativo se le denomina recuperación, pero cuando se evidencia un crecimiento positivo, se le denomina expansión.

No obstante, después de la Segunda Guerra Mundial, cuando se evidenció un crecimiento estable, que influyó en lo que muchos economistas pensaban sobre la 
forma de medir los ciclos económicos, se plantearon definiciones alternativas como las del "ciclo del crecimiento", definido por Mintz $(1974$, p. 6) como:

Un ciclo de crecimiento es una fluctuación en la actividad económica agregada, que consiste en un período de tasas relativamente altas de crecimiento que ocurren casi al mismo tiempo en muchas actividades económicas, seguido de un período de bajas tasas de crecimiento, de carácter general, que se une a la fase de alto crecimiento del siguiente ciclo.

Mintz (1974) también subraya que para el ciclo de crecimiento se sustituye la palabra "expansión" y "contracción" (utilizadas en el ciclo clásico) por "período de tasas de crecimiento relativamente altas" y "período de tasas de crecimiento relativamente bajas", en cada caso.

\subsection{Aproximaciones para fechar los ciclos}

Existen dos caminos tradicionales para fechar el origen y el fin de los ciclos económicos, la de los ciclos clásicos y la de los ciclos de crecimiento (Harding y Pagan, 2005). En el primer caso, se propone realizar la cronología de un ciclo, guiados por la presencia de puntos de quiebre en una variable continua, la cual contiene información sobre la economía en un momento del tiempo dado. Esta variable se denomina . La idea que plantea este enfoque es construir una variable binaria y aleatoria, (), que toma valores distintos según la fase del ciclo en la cual se encuentre la economía, expansión o contracción (antes o después del punto de quiebre). La técnica más utilizada es el algoritmo de Bry y Boschan (1971) que sirve para detectar máximos y mínimos locales de series mensuales, desestacionalizadas y univariadas, sujetas a ciertas reglas de censura. Los puntos identificados son considerados picos y valles que representan momentos de quiebre en la serie, y diferencian expansiones de contracciones en la actividad económica. La técnica fue expandida por Harding y Pagan (2002) para considerar datos trimestrales en lugar de mensuales.

Por el lado de los ciclos del crecimiento se puede realizar la medición de los ciclos como desviaciones con respecto a una tendencia, o como variaciones en la tasa de crecimiento de la serie1. En el primer caso, la propuesta para fechar los ciclos consiste en separar la serie en un componente de tendencia y un residuo, (). Se extrae el componente de tendencia y se denomina ciclo al residuo. De esta forma, el ciclo será entendido como las desviaciones de la variables (por lo general, el producto de la economía) con respecto a una tendencia de largo plazo. Esta aproximación también puede ser entendida como un ejercicio de hallar un pico en la

1 Para ampliar la información sobre los ciclos de crecimiento se pueden consultar los trabajos de Lucas Jr (1977) y Kydland, Prescott, y Todd (1990). 
densidad espectral de (). Existen varios filtros estadísticos que son usados para tal fin. Los más comunes son los de Hodrick-Prescott (HP), Beveridge y Nelson (1981) y en general, los de Paso de Banda (Baxter y King, 1999; Christiano y Fitzgerald, 2003). Finalmente, Harding y Pagan (2005) sugieren que la metodología de variaciones respecto a la tasa de crecimiento, podría considerarse como un caso especial de las desviaciones respecto a la tendencia de largo plazo, en la que, al suponer una tendencia estocástica en la serie, la tasa de crecimiento y sus deviaciones con respecto a un valor 'natural' dan lugar al ciclo en cada instante de tiempo.

Hamilton (2011) plantea una tipología complementaria. En términos generales, propone que el ciclo de referencia no debe ser construido con base en un solo indicador, sino que debe involucrar la observación de varios indicadores macroeconómicos. En este caso, la información de las distintas series debe ser condensada en una serie de referencia y para eso es posible acudir dos procedimientos. El primero de estos consiste en agregar las variables de interés en una sola y luego identificar los ciclos (agregar y luego fechar), mientras que el segundo consiste en identificar los ciclos y luego agregarlos (fechar y luego agregar).

\subsection{Características de los ciclos de los negocios}

Las características de las fases cíclicas pueden ser sintetizadas en tres dimensiones: amplitud, duración y asimetría:

- Amplitud: se refiere a la diferencia entre el valor mínimo del ciclo y el máximo anterior, es decir que en periodos de expansión es la ganancia (mayor producción) durante dicho periodo, mientras que en periodos de recesión la amplitud corresponderá al porcentaje de pérdida (menor producción).

- Duración: en una expansión corresponde al tiempo que trascurre desde el valle, que es el nivel más bajo de actividad económica alcanzado al final de la recesión, hasta el siguiente pico, que es el punto más alto alcanzado por la expansión de la actividad económica. Por lo mismo, la duración de una recesión corresponde al tiempo transcurrido desde un pico hasta el siguiente valle. Por consiguiente, para determinar la duración del ciclo, basta con sumar las duraciones de la recesión y la expansión.

- Asimetría: un ciclo será simétrico cuando la duración de la expansión sea igual a la duración de la contracción. Por el contrario, un ciclo será asimétrico, cuando estas dos duraciones sean diferentes. La literatura ha observado que los ciclos son asimétricos, ya que las recesiones son menos prolongadas que las expansiones. 


\subsection{Literatura relacionada}

La literatura nacional ha hecho uso, tanto de la metodología clásica, como de la basada en las tasas de crecimiento de las variables. Por ejemplo, Melo, French y Langebaek (1988), fueron los primeros en construir el ciclo de referencia de la economía colombiana basándose en un índice de difusión acumulado, para el cual utilizaron 73 series en niveles. Los autores determinan la cronología del ciclo de negocios colombiano entre 1966 y 1987. Por su parte, Arango y otros (2008) utilizan la metodología de Bry y Boschan (1971) para fechar los picos y los valles de los ciclos de negocios colombianos, comprendidos entre 1980 y 2007. Los autores concluyen que durante dicho periodo (26 años) se evidenciaron 5 ciclos de negocios completos, los cuales tuvieron en promedio 4 años de duración.

En la misma tradición de los ciclos clásicos, Alfonso y otros (2012) utilizan ambas metodologías (índice de difusión y Bry-Boschan) para estimar la cronología del ciclo de negocios colombiano entre 1975 y 2011. Para la estimación del índice de difusión acumulado utilizan 41 series. Encuentran que en ese periodo se presentaron $4 \mathrm{ci}$ clos de negocios, los cuales tuvieron una duración promedio de 6,8 años. Además, encuentran que las expansiones tienen en promedio mayor duración que las contracciones, siendo las primeras de 5,4 años mientras que las segundas de 1,3 años.

Por otro lado, el campo de los ciclos de crecimiento, ha sido examinado por estudios como los de Posada (1999), Avella y Fergusson (2004) y Arango, Arias y Flórez (2007). El primer autor analiza el ciclo económico durante el siglo XX. Explica que en la economía colombiana, en ese periodo, rara vez se presentaron caídas en el producto global, y por tanto es inapropiado estimar el ciclo económico en niveles. Sugiere entonces que un criterio para detectar las fases de auge o recesión en la economía, será comparar el ingreso observado, en un periodo dado, con el ingreso permanente. De esta forma, la economía estará en auge, si durante dos años consecutivos o más, el producto real observado supera el permanente (conversamente se encontrará en recesión si durante dos años o más el producto real es inferior al permanente). Ahora bien, para obtener una medida del producto permanente, el autor utiliza el filtro de Hodrick-Prescott (HP). Esta aproximación no está libre de críticas, a su vez, y su uso en el presente contexto bien podría empeorar la estimación de los ciclos, llevando a sesgos innecesarios y a la aparición de correlaciones espurias entre distintas variables cíclicas, provenientes de series macroeconómicas, tal y como lo señala Canova (1998).

Avella y Fergusson (2004) exploran los ciclos económicos a lo largo del siglo XX, desde una perspectiva teórica, y realizan también un análisis de las correlaciones entre ciclos económicos de diferentes países. Los autores estudian la correlación 
entre el ciclo colombiano y el estadounidense. Para esto último, optan por distinguir el componente "permanente" y las desviaciones "transitorias" de las series de ambas economías, por medio de la aplicación de los filtros de Hodrick y Prescott, y de Baxter y King. Los resultados de la investigación revelan la ausencia de correlaciones estadísticamente significativas entre los ciclos económicos de Estados Unidos y Colombia.

Por otra parte, Arango, Arias y Flórez (2007) estiman las fechas de los ciclos de crecimiento de Colombia entre 1980 y 2007, mediante la metodología de Boschan y Ebanks (1978) conocida como "Phase Averange Trend" (PAT) y comparan las características de los ciclos de crecimiento con las que surgen al calcular los ciclos utilizando el filtro de HP y el método de tendencia lineal local. Concluyen que la utilización del PAT logra estimaciones muy similares a las encontradas con la utilización del filtro de HP y que los ciclos de crecimiento son, por lo general, más pequeños, frecuentes, menos variables y mucho más simétricos que los ciclos de los negocios.

En este documento se utiliza la metodología del ciclo clásico, para evitar las múltiples críticas que ha recibido la utilización de técnicas basadas en filtros, para tal fin (Canova, 1998). Estas críticas se apoyan en que la introducción de los filtros modifica de forma significativa el comportamiento original de las variables bajo estudio, y puede llevar a la aparición de comovimientos espurios entre distintas series, o a sesgos en la estimación de los ciclos, cuando los parámetros del filtro no se especifican de forma correcta. Aporta a la literatura nacional, puesto que no existen estudios que construyan un ciclo de referencia partiendo de una identificación clásica de los ciclos en el esquema de "fechar y luego agregar", ni estudios que permitan medir el grado de sincronización de los mismos con variables proxy de las políticas de estabilización nacionales.

\section{METODOLOGÍA}

\subsection{Datos}

Las variables empleadas para construir el ciclo de los negocios son: un índice de Producción industrial, un índice de Producción industrial excluyendo el café y un índice de ventas excluyendo el café. Todas en términos trimestrales desde marzo de 1990 hasta junio de 2013, es decir 93 observaciones por cada variable. Estas series fueron tomadas de Datastream Internacional. Para el ciclo de política fiscal se utiliza el consumo del gobierno, a precios del año 2000, y para el ciclo de política monetaria se utiliza la tasa de captación, que las instituciones depositarias reportan periódicamente, pagadas por los Certificados de Depósito a Término Fijo (CDT). En este caso, las fuentes son el Departamento Administrativo Nacional de Estadística en el primer caso y la página web del Banco de la República en el segundo. 


\subsection{Cronología de los ciclos para cada variable}

Se utiliza la modificación de la metodología de Bry y Boschan (1971), propuesta por Harding y Pagan $(2002,2006)$ para fechar los ciclos de varias series relacionadas con el desempeño económico colombiano. Se utilizó el software estadístico R para llevar a cabo las estimaciones, en particular el paquete 'BCDating'.

La metodología de Bry y Boschan (1971) se resume en que una observación es considerada una candidata para ser un pico de una variable, si y candidata para ser un valle si. Para garantizar una identificación mayor se imponen una serie de restricciones adicionales:

- Los picos y los valles deben alternarse. En caso de ruptura el menor de los dos picos es eliminado.

- Un pico (valle) debe ser mayor (menor) que el valle (pico) previo.

- La longitud mínima entre pico y valle es de 2 trimestres.

- Los puntos de quiebre que ocurran entre los primeros dos o los últimos dos trimestres son eliminados.

- Picos (valles) al comienzo o al final que son más bajos (mayores) que los valores (finales) son eliminados.

\subsection{Agregación de los ciclos en un ciclo de referencia único}

Por otro lado, la etapa de agregación de los ciclos individuales en uno de referencia sigue la propuesta de Canova y Schlaepfer (2012). Así, considérense las series de indicadores donde. Con los puntos de quiebre que se computan para cada serie de forma individual, se calcula en cada la distancia entre trimestres al pico más cercano y se crea de esta forma un nuevo vector estocástico denominado. De la misma forma, se calcula la distancia en trimestres al valle más cercano y se crea otro vector de series denominado. Después estas dos series se suma en y se procede a determinar las fechas en las que el agregado encuentra un punto mínimo. Intuitivamente, valores bajos de indican que varias series se encuentran cercanas a un pico (valle) en el momento . Por tanto, estos mínimos locales son candidatos a picos y valles en el ciclo de referencia. Una vez estos candidatos son identificados se imponen las mismas reglas de censura descritas antes. La agregación de y se realiza a través de la media simple.

\subsection{Estimación del grado de sincronización}

El método descrito en el primer apartado de esta sección sirve para construir el ciclo de negocios de Colombia, el ciclo de política monetaria y el ciclo de política fiscal. 
El análisis de sincronización se lleva a cabo mediante el estadístico de concordancia propuesto por Harding y Pagan (2006):

$\hat{\imath}=T^{-1} \sum_{\mathrm{t}=1}^{\mathrm{T}} \mathrm{S}_{\mathrm{xt}} \mathrm{S}_{\mathrm{yt}}+\sum_{\mathrm{t}=1}^{\mathrm{T}}\left(1-\mathrm{S}_{x t}\right)\left(1-\mathrm{S}_{x t}\right)$

Donde xt es la serie con la información sobre la actividad real colombiana y $y_{t}$ es la serie con la información sobre la política de estabilización. $\mathrm{S}_{y t}$ y $\mathrm{S}_{x t}$ son variables binarias que toman el valor de 1 o 0 , según si variable se encuentra en una fase de expansión o contracción. El índice de concordancia mide la proporción del tiempo en que dos series se encuentran en la misma fase; un valor de 1 implica que los dos ciclos están en la misma fase todo el tiempo.

Un análisis adicional de sincronización consiste en examinar los patrones visuales de picos y valles con el fin de establecer la secuencia en que la economía entra y sale de cada fase.

\section{RESULTADOS}

\subsection{Ciclos Individuales y Ciclo de Referencia}

Como se expuso en la sección 2.1. el ciclo de negocios de Colombia se construyó con 3 variables reales: un índice de Producción Industrial (IPI 1), un índice de Producción Industrial excluyendo el café (IPI 2) y un índice de Ventas Industriales excluyendo el café (ISI por sus siglas en ingles).

Se considera que dichas variables capturan el ciclo de negocios de la economía colombiana, ya que siguiendo a Hirschman (1983) y Currie $(1968,1993)$, la industria es el sector que presenta mayores eslabonamientos productivos hacia atrás. Esta idea sugiere que desde la producción industrial se mueven muchos sectores satélites, por lo que si se registra un crecimiento o una ralentización de la misma, se verán afectados, directa o indirectamente, otros sectores de la economía. También se seleccionaron por ser los únicos índices disponibles con la frecuencia necesaria, directamente relacionados con la actividad industrial, en la base de datos. En futuras investigaciones, se puede recurrir a la inclusión de otras variables reales, por ejemplo series de desempleo, o de actividad económica externa.

A continuación se realiza una descripción de los ciclos individuales, asociados con cada una de las variables utilizadas en la construcción del ciclo de referencia de la economía colombiana. La primera variable es el Índice de Producción Industrial trimestral, el cual se puede observar en la Gráfica 1. Las franjas grises indican que durante ese periodo se evidenció una contracción del índice, mientras que los 
espacios en blanco indican una expansión. En la Tabla 1, se presenta un resumen más detallado del comportamiento descrito de la variable.

Durante este periodo se observan 4 ciclos completos, de pico a pico, es decir 5 expansiones y 5 recesiones. La duración promedio de las expansiones es de 13 trimestres, mientras que la duración promedio de las recesiones es de 2,8 trimestres, lo que da claros indicios de asimetría en el ciclo de este índice. El ciclo completo de mayor duración para esta variable (de pico a pico), fue el que se presentó entre octubre de 2001 y octubre del 2008 (28 trimestres), mientras que el más corto se presentó entre marzo de 1996 y marzo de 1998 (8 trimestres).

Gráfica 1. Comportamiento del Índice de Producción Industrial

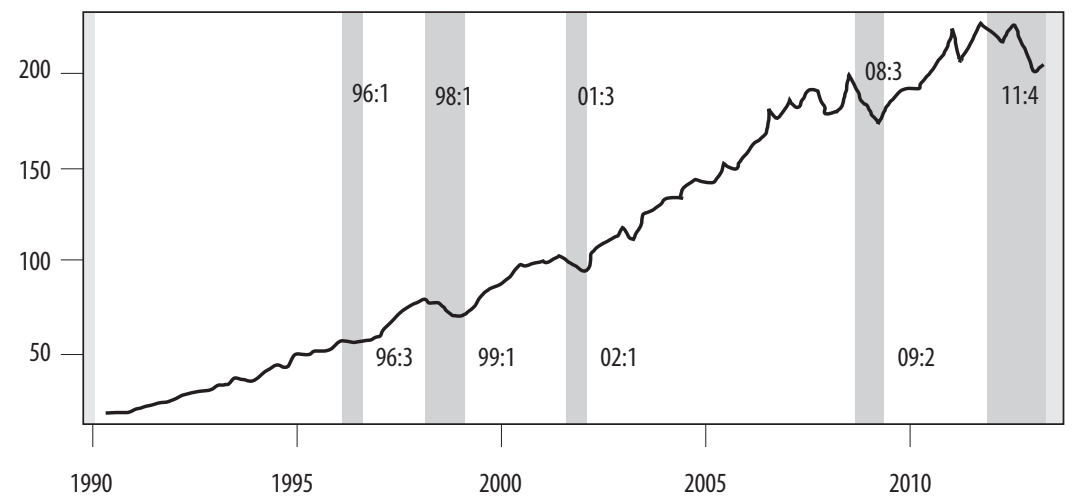

Nota: Las áreas sombreadas corresponden con periodos de contracción en la variable, de acuerdo con la metodología clásica. Las áreas blancas con periodos de expansión.

Fuente: elaboración propia.

Tabla 1. Fases de expansión y recesión del Índice de Producción Industrial

\begin{tabular}{|l|c|c|c|c|c|c|}
\hline \multicolumn{2}{|c|}{ ÍNDICE DE PRODUCCIÓN INDUSTRIAL } \\
\hline & Empieza & Termina & $\begin{array}{c}\text { Duración } \\
\text { trimestres) }\end{array}$ & $\begin{array}{c}\text { Nivel en el que } \\
\text { Empieza }\end{array}$ & $\begin{array}{c}\text { Nivel en el que } \\
\text { Termina }\end{array}$ & Amplitud \\
\hline Expansión & $<\mathrm{NA}>$ & $1996 \mathrm{Q} 1$ & NA & NA & 57 & NA \\
\hline Recesión & $1996 \mathrm{Q} 1$ & 199603 & 2 & 57 & 56 & 0,9 \\
\hline Expansión & $1996 \mathrm{Q} 3$ & $1998 \mathrm{Q} 1$ & 6 & 56 & 79 & 22,8 \\
\hline Recesión & 199801 & 199901 & 4 & 79 & 70 & 9,4 \\
\hline Expansión & 199901 & 200103 & 10 & 70 & 104 & 34,9 \\
\hline
\end{tabular}




\begin{tabular}{|l|c|c|c|c|c|c|}
\hline \multicolumn{2}{|c|}{ ÍNDICE DE PRODUCCIÓN INDUSTRIAL } \\
\hline & Empieza & Termina & $\begin{array}{c}\text { Duración } \\
\text { (trimestres) }\end{array}$ & $\begin{array}{c}\text { Nivel en el que } \\
\text { Empieza }\end{array}$ & $\begin{array}{c}\text { Nivel en el que } \\
\text { Termina }\end{array}$ & Amplitud \\
\hline Recesión & $2001 \mathrm{Q} 3$ & $2002 \mathrm{Q} 1$ & 2 & 104 & 93 & 11,1 \\
\hline Expansión & $2002 \mathrm{Q} 1$ & $2008 \mathrm{Q} 3$ & 26 & 93 & 201 & 107,9 \\
\hline Recesión & $2008 \mathrm{Q} 3$ & $2009 \mathrm{Q} 2$ & 3 & 201 & 173 & 28,0 \\
\hline Expansión & $2009 \mathrm{Q} 2$ & $2011 \mathrm{Q} 4$ & 10 & 173 & 229 & 56,2 \\
\hline Recesión & $2011 \mathrm{Q} 4$ & $<\mathrm{NA}>$ & NA & 229 & NA & NA \\
\hline
\end{tabular}

Nota: Fechas de inicio de finalización de cada fase del ciclo en la variable. Se presenta también la duración y amplitud, que sirven para caracterizar los ciclos.

Fuente: elaboración propia.

La segunda variable que se utilizó para la construcción del ciclo de negocios fue el índice de Producción Industrial excluyendo el café (Gráfica 2 y Tabla 2).

Gráfica 2. Comportamiento del Índice de Producción Industrial excluyendo el café

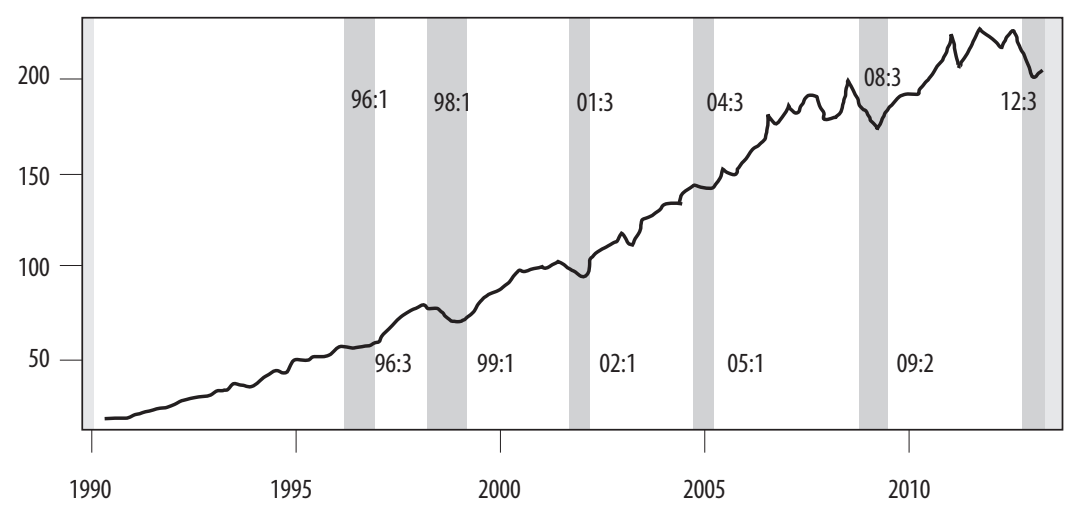

Nota: Las áreas sombreadas corresponden con periodos de contracción en la variable, de acuerdo con la metodología clásica. Las áreas blancas con periodos de expansión.

Fuente: elaboración propia.

Como se puede observar, durante este periodo se presentaron 6 recesiones y 6 expansiones, es decir 5 ciclos completos de pico a pico. La duración promedio de las fases de expansión es de 10,4 trimestres mientras que la de las recesiones es de 2,8. Por otra parte, los ciclos completos más largos de este índice (de pico a pico) son los que se presentan entre octubre de 2004 y octubre de 2008, y el que se presenta entre octubre de 2008 y octubre de 2012. Mientras que el más corto se registró entre marzo de 1996 y marzo de 1998. 
Ciclo de los negocios en Colombia: el papel de la política de estabilización

Tabla 2. Fases de expansión y recesión del Î́ndice de Producción Industrial excluyendo el café

\begin{tabular}{|l|c|c|c|c|c|c|}
\hline \multicolumn{2}{|c|}{ ÍNDICE DE PRODUCCIÓN INDUSTRIAL EXCLUYENDO CAFÉ } \\
\hline & Empieza & Termina & $\begin{array}{c}\text { Duración } \\
\text { (trimestres) }\end{array}$ & $\begin{array}{c}\text { Nivel en el que } \\
\text { Empieza }\end{array}$ & $\begin{array}{c}\text { Nivel en el que } \\
\text { Termina }\end{array}$ & Amplitud \\
\hline Expansión & $<\mathrm{NA}>$ & $1996 \mathrm{Q} 1$ & $\mathrm{NA}$ & $\mathrm{NA}$ & 56 & NA \\
\hline Recesión & $1996 \mathrm{Q} 1$ & $1996 \mathrm{Q} 4$ & 3 & 56 & 55 & 0,9 \\
\hline Expansión & $1996 \mathrm{Q} 4$ & $1998 \mathrm{Q} 1$ & 5 & 55 & 76 & 21,7 \\
\hline Recesión & $1998 \mathrm{Q} 1$ & $1999 \mathrm{Q} 1$ & 4 & 76 & 67 & 9,2 \\
\hline Expansión & $1999 \mathrm{Q} 1$ & $2001 \mathrm{Q} 3$ & 10 & 67 & 105 & 37,4 \\
\hline Recesión & $2001 \mathrm{Q} 3$ & $2002 \mathrm{Q} 1$ & 2 & 105 & 93 & 11,7 \\
\hline Expansión & $2002 \mathrm{Q} 1$ & $2004 \mathrm{Q} 3$ & 10 & 93 & 143 & 49,9 \\
\hline Recesión & $2004 \mathrm{Q} 3$ & $2005 \mathrm{Q} 1$ & 2 & 143 & 139 & 4,0 \\
\hline Expansión & $2005 \mathrm{Q} 1$ & $2008 \mathrm{Q} 3$ & 14 & 139 & 201 & 61,7 \\
\hline Recesión & $2008 \mathrm{Q} 3$ & $2009 \mathrm{Q} 2$ & 3 & 201 & 173 & 27,4 \\
\hline Expansión & $2009 \mathrm{Q} 2$ & $2012 \mathrm{Q} 3$ & 13 & 173 & 228 & 55,1 \\
\hline Recesión & $2012 \mathrm{Q} 3$ & $<\mathrm{NA}>$ & $\mathrm{NA}$ & 228 & NA & NA \\
\hline
\end{tabular}

Nota: Fechas de inicio de finalización de cada fase del ciclo en la variable. Se presenta también la duración y amplitud, que sirven para caracterizar los ciclos.

Fuente: elaboración propia.

La tercera y última variable es el Índice de Ventas Industriales excluyendo la producción de café (ISI), ver Gráfica 3 y Tabla 3. Esta variable describe 4 ciclos completos, es decir, 5 expansiones y 5 recesiones, con duración promedio de 12 y 3 trimestres respectivamente. Como se puede notar, también existe asimetría en el ciclo del índice de ventas industriales. En este caso, el ciclo completo más largo se presenta entre diciembre de 2002 y octubre de 2008, mientras que el más corto se da entre diciembre de 2011 y diciembre de 2012.

Gráfica 3. Comportamiento del Índice de Ventas Industriales

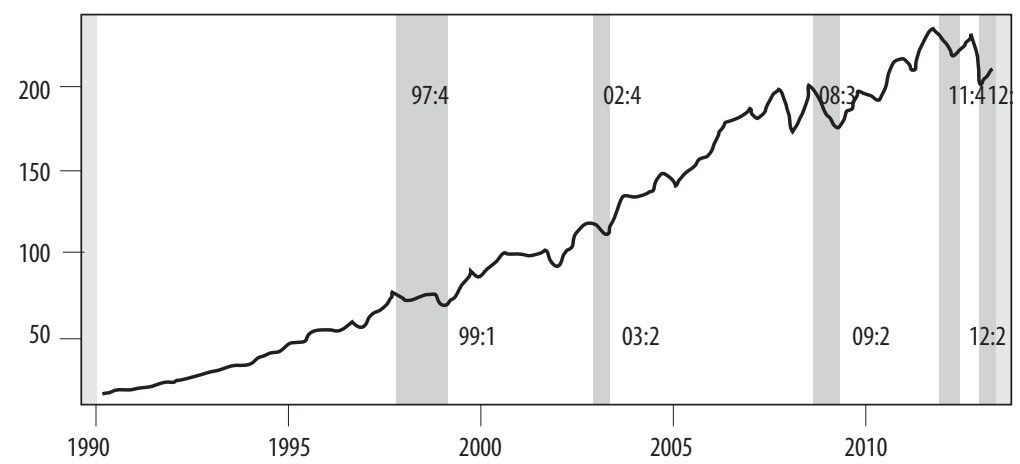

Nota: Las áreas sombreadas corresponden con periodos de contracción en la variable, de acuerdo con la metodología clásica.

Las áreas blancas con periodos de expansión.

Fuente: elaboración propia. 
Jorge Mario Uribe - Karol Carbonell

Tabla 3. Fases de expansión y recesión del Índice de Ventas Industriales excluyendo el café

\begin{tabular}{|l|c|c|c|c|c|c|}
\hline \multicolumn{2}{|c|}{ ÍNDICE DE VENTAS INDUSTRIALES EXCLUYENDO CAFÉ } \\
\hline & Empieza & Termina & $\begin{array}{c}\text { Duración } \\
\text { (trimestres) }\end{array}$ & $\begin{array}{c}\text { Nivel en el que } \\
\text { Empieza }\end{array}$ & $\begin{array}{c}\text { Nivel en el que } \\
\text { Termina }\end{array}$ & Amplitud \\
\hline Expansión & $<\mathrm{NA}>$ & $1997 \mathrm{Q} 4$ & $\mathrm{NA}$ & $\mathrm{NA}$ & 77 & NA \\
\hline Recesión & $1997 \mathrm{Q} 4$ & $1999 \mathrm{Q} 1$ & 5 & 77 & 70 & 7,1 \\
\hline Expansión & $1999 \mathrm{Q} 1$ & $2002 \mathrm{Q} 4$ & 15 & 70 & 119 & 49,8 \\
\hline Recesión & $2002 \mathrm{Q} 4$ & $2003 \mathrm{Q} 2$ & 2 & 119 & 110 & 9,5 \\
\hline Expansión & $2003 \mathrm{Q} 2$ & $2008 \mathrm{Q} 3$ & 21 & 110 & 202 & 92,3 \\
\hline Recesión & $2008 \mathrm{Q} 3$ & $2009 \mathrm{Q} 2$ & 3 & 202 & 175 & 27,5 \\
\hline Expansión & $2009 \mathrm{Q} 2$ & $2011 \mathrm{Q} 4$ & 10 & 175 & 236 & 61,4 \\
\hline Recesión & $2011 \mathrm{Q} 4$ & $2012 \mathrm{Q} 2$ & 2 & 236 & 218 & 18,0 \\
\hline Expansión & $2012 \mathrm{Q} 2$ & $2012 \mathrm{Q} 4$ & 2 & 218 & 233 & 15,0 \\
\hline Recesión & $2012 \mathrm{Q} 4$ & $<\mathrm{NA}>$ & $\mathrm{NA}$ & 233 & $\mathrm{NA}$ & NA \\
\hline
\end{tabular}

Nota: Fechas de inicio de finalización de cada fase del ciclo en la variable. Se presenta también la duración y amplitud, que sirven para caracterizar los ciclos.

Fuente: elaboración propia

Después de agregar los ciclos de las tres variables, por medio de la metodología expuesta, se muestra en la Tabla 4 la cronología del ciclo de los negocios de Colombia entre marzo de 1990 y junio de 2013 y se le compara con las propuestas en estudios anteriores, en la Tabla 5.

Durante marzo de 1990 y junio de 2013 se evidencian 3 ciclos completos de pico a pico, el primero entre marzo de 1996 y marzo de 1998, el segundo entre marzo de 1998 y octubre de 2001 y el tercero entre octubre de 2001 y octubre del 2008. Se puede ver que esta metodología logra captar la recesión de 1998 - 1999 y la de 2008 - 2009. No existen estadísticos multivariados de amplitud por lo cual no se reportan en la Tabla.

Tabla 4. Fases de expansión y recesión del ciclo de negocios de Colombia

\begin{tabular}{|l|c|c|}
\hline \multicolumn{3}{|c|}{ CICLO DE NEGOCIOS DE COLOMBIA } \\
\hline Expansión & Empieza & Termina \\
\hline Recesión & $<\mathrm{NA}>$ & $1996 \mathrm{Q} 1$ \\
\hline Expansión & $1996 \mathrm{Q} 1$ & $1996 \mathrm{Q} 4$ \\
\hline Recesión & $1996 \mathrm{Q} 4$ & $1998 \mathrm{Q} 1$ \\
\hline Expansión & $1998 \mathrm{Q} 1$ & $1999 \mathrm{Q} 1$ \\
\hline Recesión & $1999 \mathrm{O} 1$ & $2001 \mathrm{Q} 3$ \\
\hline Expansión & $2001 \mathrm{Q} 3$ & $2002 \mathrm{Q} 1$ \\
\hline Recesión & $2002 \mathrm{O} 1$ & $2008 \mathrm{Q} 3$ \\
\hline Expansión & $2008 \mathrm{Q} 3$ & $2011 \mathrm{Q} 4$ \\
\hline
\end{tabular}

Nota: Fechas de inicio de finalización de cada fase del ciclo en la variable. Se presenta también la duración y amplitud, que sirven para caracterizar los ciclos.

Fuente: elaboración propia. 
Tabla 5. Comparación entre cronologías propuestas para el ciclo de negocios de Colombia.

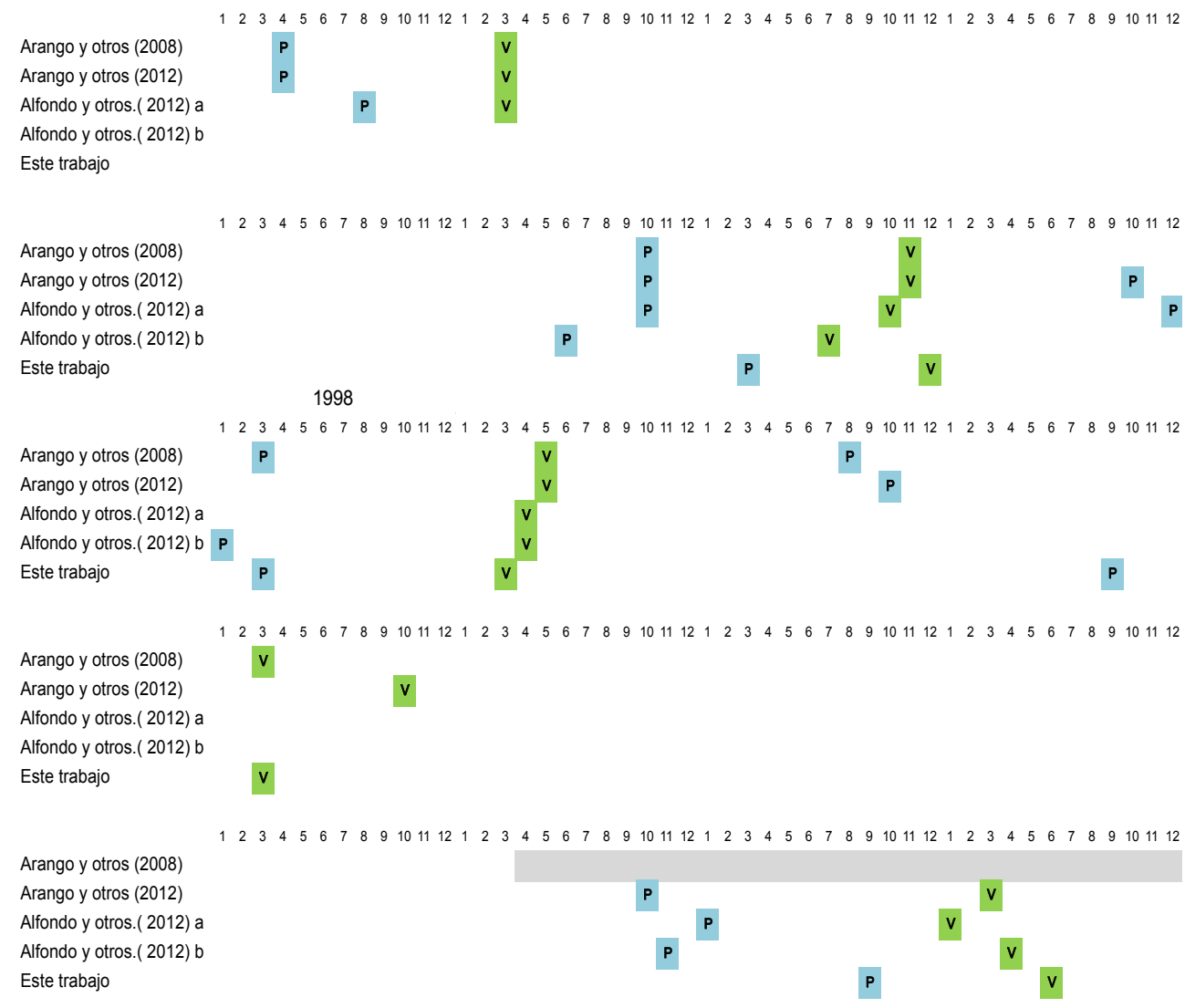

$\begin{array}{llllllllllllllllllllllllllllllllllllllllllllllll}1 & 2 & 3 & 4 & 5 & 6 & 7 & 8 & 9 & 10 & 11 & 12 & 1 & 2 & 3 & 4 & 5 & 6 & 7 & 8 & 9 & 10 & 11 & 12 & 1 & 2 & 3 & 4 & 5 & 6 & 7 & 8 & 9 & 10 & 11 & 12 & 1 & 2 & 3 & 4 & 5 & 6 & 7 & 8 & 9 & 10 & 11 & 12\end{array}$

Arango y otros (2008)

Arango y otros (2012)

Alfondo y otros.( 2012) a

Alfondo y otros.( 2012) b

Este trabajo

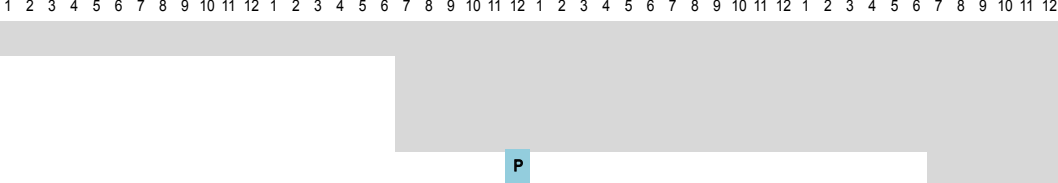

Nota: la letra $V$ significa valle y la letra $P$ significa pico. Los espacios en gris indican que para esos periodos no hay estimaciones. En la investigación de Alfonso y otros (2012) se estimaron 3 cronologías del ciclo de negocios, la primera con base en el Índice de Producción Industrial, la segunda en un Índice de Difusión Acumulado (a) y la tercera estimada mediante el algoritmo de Leamer (b). Se reportan las últimas dos a modo de comparación en la tabla.

Fuente: elaboración propia

En cuanto a las políticas de estabilización se tiene lo siguiente. Para la política fiscal se eligió el consumo del gobierno trimestral. El comportamiento de esta variable se presenta en la Gráfica 4, en donde se evidencian 3 fases de recesión y 4 de expansión. En la Tabla 6, se realiza un resumen del ciclo de política fiscal. 
Gráfica 4. Comportamiento del Consumo del Gobierno

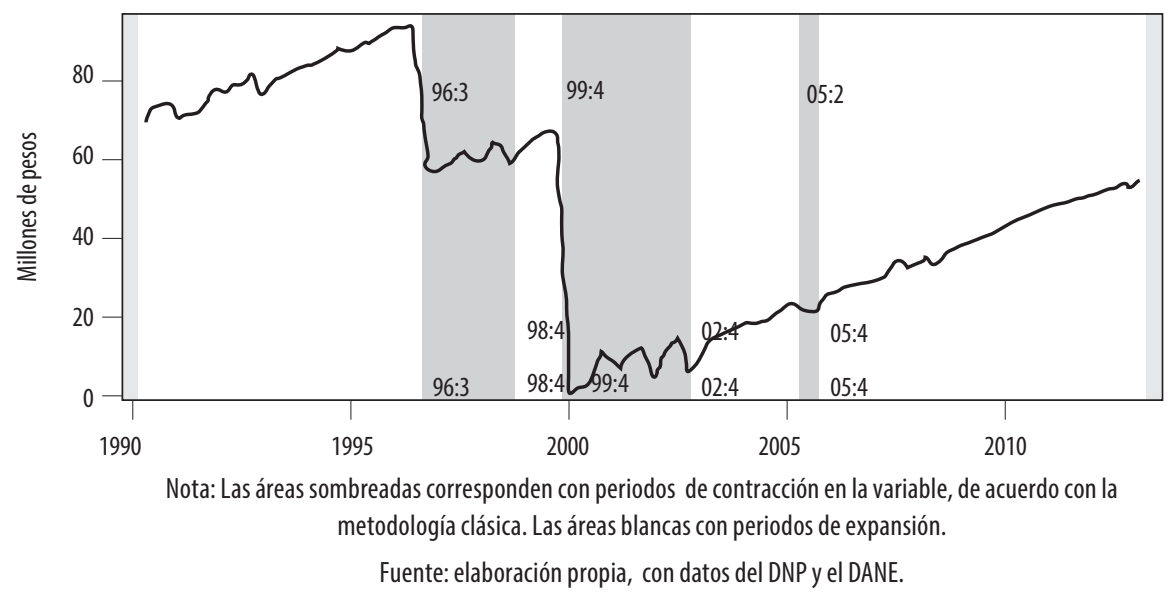

Tabla 6. Fases de expansión y recesión del Consumo Gubernamental

\begin{tabular}{|l|c|c|c|c|c|c|}
\hline & Empieza & Termina & $\begin{array}{c}\text { Duración } \\
\text { (trimestres) }\end{array}$ & $\begin{array}{c}\text { Nivel en el que } \\
\text { Empieza }\end{array}$ & $\begin{array}{c}\text { Nivel en el que } \\
\text { Termina }\end{array}$ & Amplitud \\
\hline Expansión & $<\mathrm{NA}>$ & $1996 \mathrm{Q} 3$ & NA & NA & 94 & NA \\
\hline Recesión & $1996 \mathrm{Q} 3$ & $1998 \mathrm{Q} 4$ & 9 & 94 & 59 & 35 \\
\hline Expansión & $1998 \mathrm{Q} 4$ & $1999 \mathrm{Q} 4$ & 4 & 59 & 68 & 9 \\
\hline Recesión & $1999 \mathrm{Q} 4$ & $2002 \mathrm{Q} 4$ & 12 & 68 & 6 & 62 \\
\hline Expansión & $2002 \mathrm{Q} 4$ & $2005 \mathrm{O} 2$ & 10 & 6 & 25 & 19 \\
\hline Recesión & $2005 \mathrm{Q} 2$ & $2005 \mathrm{Q} 4$ & 2 & 25 & 22 & 3 \\
\hline Expansión & $2005 \mathrm{Q} 4$ & $<\mathrm{NA}>$ & NA & 22 & NA & NA \\
\hline
\end{tabular}

Nota: Fechas de inicio de finalización de cada fase del ciclo en la variable. Se presenta también la duración y amplitud, que sirven para caracterizar los ciclos.

Fuente: elaboración propia.

Durante junio de 1990 y junio de 2013, se evidenciaron 2 ciclos completos. La duración promedio de las expansiones fue de 7 trimestres, mientras que la duración promedio de las recesiones fue de 7,7 trimestres. El ciclo completo más largo se presentó entre diciembre de 1999 y junio de 2005, mientras que el ciclo más corto tuvo lugar entre octubre de 1996 y diciembre de 1999. Es importante aclarar que cuando el ciclo de la política fiscal (consumo del gobierno) se encuentra en 'expansión', indica que se está realizando una política expansiva (aumenta el consumo del gobierno), mientras que cuando está en 'recesión', se estaría realizando una política contractiva (disminuye el consumo del gobierno). 
Como proxy de las condiciones de liquidez, y por ende de la política monetaria, en la economía, se empleó la tasa de interés de captación de los Certificados de Depósito a Término Fijo (CDTs) a diferentes plazos. Esta variable presenta 8 ciclos completos de pico a pico, dentro de los cuales se cuentan 9 expansiones y 9 recesiones (ver Gráfica 5 y Tabla 8). El ciclo más largo se documenta entre diciembre de 2003 y diciembre de 2008, mientras que los más cortos se presentan de octubre de 1991 a diciembre de 1992, y de diciembre de 1996 a marzo de 1996. La duración promedio de las expansiones es de 4,6 trimestres, mientras que la de las recesiones es de 5,8 trimestres. Cabe aclarar que cuando se habla de expansiones en esta variable, la tasa de interés de captación aumenta, siguiendo una reducción de la liquidez en el mercado, algunas veces como consecuencia de un incremento en la tasa de referencia de la economía. Por otra parte, cuando esta variable entra en una fase contractiva, la tasa disminuye, lo cual corresponde con una política monetaria expansiva.

Gráfica 5. Comportamiento de la Tasa de Captación

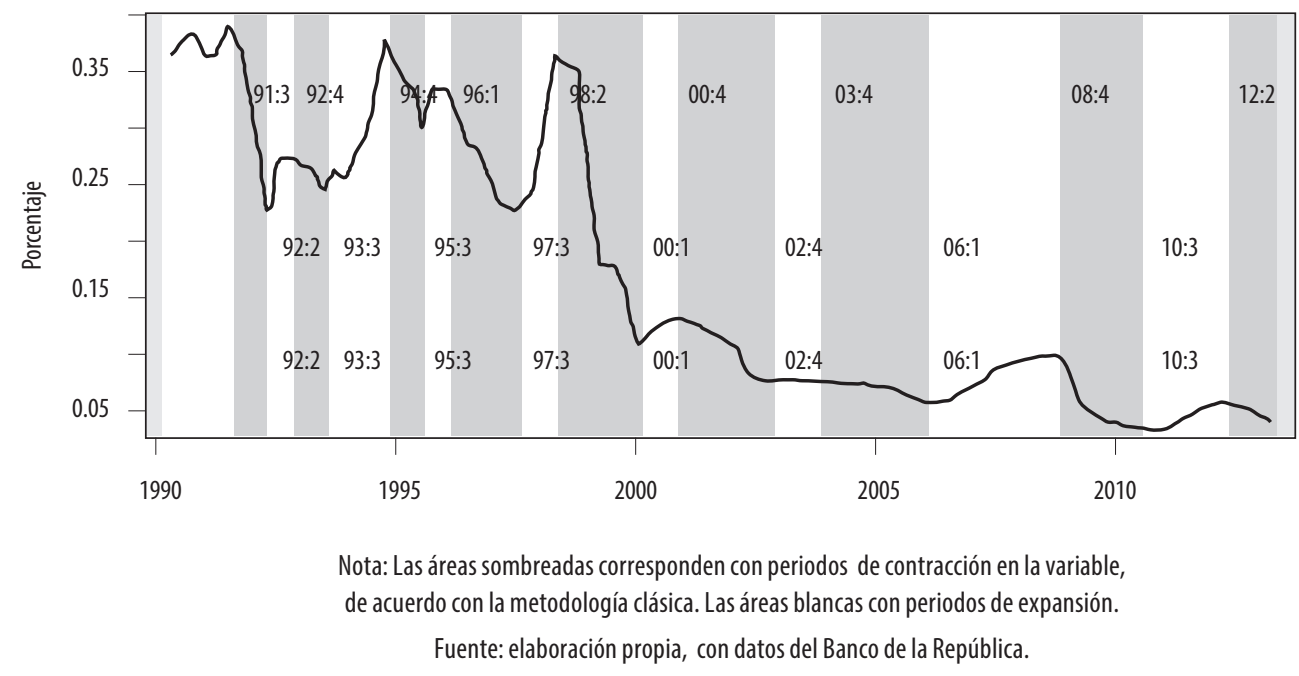

A continuación se examina si las condiciones monetarias y fiscales han tenido el comportamiento esperado, dado su papel como estabilizadoras de la economía. Es decir, si tienen un carácter contra-cíclico, que concuerde con un intento de las autoridades monetarias y fiscales de atenuar las oscilaciones en el tiempo del producto. 
Jorge Mario Uribe - Karol Carbonell

Tabla 7. Fases de expansión y recesión de la Tasa de Interés de Captación los CDT

\begin{tabular}{|c|c|c|c|c|c|c|}
\hline \multicolumn{7}{|c|}{ CICLO POLÍTICA MONETARIA } \\
\hline & Empieza & Termina & $\begin{array}{c}\text { Duración } \\
\text { (trimestres) }\end{array}$ & $\begin{array}{c}\text { Nivel en el que } \\
\text { Empieza }\end{array}$ & $\begin{array}{c}\text { Nivel en el que } \\
\text { Termina }\end{array}$ & Amplitud \\
\hline Expansión & $<\mathrm{NA}>$ & 1991Q3 & NA & NA & 0,3927 & NA \\
\hline Recesión & 199103 & 1992Q2 & 3 & 0,3927 & 0,2275 & 0,2 \\
\hline Expansión & 1992Q2 & 1992Q4 & 2 & 0,2275 & 0,2742 & 0,0 \\
\hline Recesión & 1992Q4 & 199303 & 3 & 0,2742 & 0,2437 & 0,0 \\
\hline Expansión & 199303 & 1994Q4 & 5 & 0,2437 & 0,379 & 0,1 \\
\hline Recesión & 1994Q4 & 199503 & 3 & 0,379 & 0,3008 & 0,1 \\
\hline Expansión & 199503 & 199601 & 2 & 0,3008 & 0,3364 & 0,0 \\
\hline Recesión & 199601 & 1997Q3 & 6 & 0,3364 & 0,2285 & 0,1 \\
\hline Expansión & 1997Q3 & 199802 & 3 & 0,2285 & 0,3656 & 0,1 \\
\hline Recesión & 1998Q2 & 200001 & 7 & 0,3656 & 0,109 & 0,3 \\
\hline Expansión & 200001 & 200004 & 3 & 0,109 & 0,1328 & 0,0 \\
\hline Recesión & 200004 & $2002 \mathrm{Q} 4$ & 8 & 0,1328 & 0,0773 & 0,1 \\
\hline Expansión & 2002Q4 & 200304 & 4 & 0,0773 & 0,0795 & 0,0 \\
\hline Recesión & 200304 & 200601 & 9 & 0,0795 & 0,0597 & 0,0 \\
\hline Expansión & 200601 & 200804 & 11 & 0,0597 & 0,1012 & 0,0 \\
\hline Recesión & 2008Q4 & 201003 & 7 & 0,1012 & 0,0347 & 0,1 \\
\hline Expansión & 201003 & $2012 \mathrm{Q} 2$ & 7 & 0,0347 & 0,0545 & 0,0 \\
\hline Recesión & $2012 \mathrm{Q} 2$ & $<\mathrm{NA}>$ & NA & 0,0545 & NA & NA \\
\hline
\end{tabular}

Fechas de inicio de finalización de cada fase del ciclo en la variable. Se presenta también la duración y amplitud, que sirven para caracterizar los ciclos.

Fuente: elaboración propia. Nota:

\subsection{Sincronización con variables de política}

Como se dijo en la sección de metodología el estadístico de concordancia mide la proporción del tiempo en el que dos series se encuentran en la misma fase (expansiva o recesiva), es decir que si el estadístico es 1, quiere decir que las dos series se encuentran en la misma fase todo el tiempo, mientras que si es de 0 indica que las dos series se encuentran en diferente fase todo el tiempo. Por lo mismo, un estadístico de concordancia de 0,5 se podría interpretar como un escenario en el cual 50 por ciento de las veces las variables se encontraron en la misma fase, o se podría decir que el 50 por ciento de las veces las series se encontraban en diferente fase, luego es señal de no correlación entre los ciclos. 
Se espera que las variables de política se comporten de manera contracíclica, para que actúen como agentes estabilizadores de las variables reales. Esto es, cuando la economía se encuentre en tiempos difíciles, la política debería de ser expansiva (aumentando el gasto del gobierno y disminuyendo la tasa de interés). Por el contrario, si la economía se encuentra en tiempos de bonanza, esta debería de ser contractiva (disminuyendo el gasto gubernamental o aumentado la tasa de interés).

Esto sugiere que el estadístico de concordancia entre la política fiscal y el ciclo de negocios de Colombia debería tender a 0 , mientras que entre el ciclo de política monetaria y ciclo de negocios colombiano, el estadístico debería tender a 1.

En la Tabla 8 se encuentra la cronología del ciclo de la política fiscal y del ciclo de los negocios. De la misma forma en la Tabla 9 se encuentra la cronología del ciclo de política monetaria y el ciclo de negocios de Colombia.

El estadístico de Harding y Pagan entre la variable fiscal y el ciclo de negocios para el periodo analizado es 0,69, esto indica que el 69 por ciento de las veces ambas series se encontraban en la misma fase. Es decir que cuando el ciclo de negocios estaba en auge, la política fiscal fue expansiva, mientras que cuando estaba en recesión la misma tendió a ser contractiva.

Por otro lado, el estadístico que mide la sincronización entre la variable de política monetaria y el ciclo de los negocios, para el periodo analizado, asciende a 0,57. Es decir, aproximadamente el 57 por ciento de las veces, las dos series se encontraron en la misma fase. Este estadístico da cuenta de cierta independencia entre el ciclo de la tasa de interés de mercado y el ciclo de los negocios (ya que es cercano a 0,5). Se podría concluir entonces que, o bien la política monetaria es contracíclica pero no se trasmite a la tasa de interés de mercado, o la misma no es contracíclica, como se espera teóricamente. En todo caso, se presenta evidencia de un alcance limitado de la variable monetaria, encarnada en la tasa de interés, para actuar como potencial estabilizador de los ciclos de la economía.

Al comportamiento procíclico documentado en la política fiscal, se le conoce en la literatura como el fenómeno "when it rains, it pours". Los hallazgos al respecto, para la economía colombiana, concuerdan con los de Kaminsky, Reinhart y Végh (2004), quienes documentan que las economías emergentes, por lo general, siguen políticas fiscales procíclicas, contrario a lo que sucede en los países industrializados.

Al respecto Reinhart y Reinhart (2009) señalan que la falta de acceso de las economías emergentes a los mercados mundiales de capitales durante periodos turbulentos, puede menguar la capacidad que tiene el gobierno de realizar políticas en dirección contraria al ciclo. 
Por otra parte, siguiendo a Spiegel (2007), entre las fuentes de ingreso del gobierno están los impuestos o la recaudación tributaria, que aumentan durante periodos de crecimiento económico de forma paralela al crecimiento de las rentas. Aunque teóricamente la financiación externa debería diversificar las fuentes de ingreso, las imperfecciones de los mercados de capitales y los ciclos de la ayuda exterior, hacen que los flujos de capital se comporten de forma procíclica, potenciando el sesgo de las cuentas fiscales.

Adicionalmente, los mercados de capitales son imperfectos y algunos países, sobretodo los más endeudados y con mayores necesidades de liquidez, se ven en ocasiones incapacitados para pedir prestado, por lo que se ven obligados a emprender una política procíclica, sobre todo en épocas de recesión general.

Por otro lado, y apoyando la idea anterior, Ocampo (2011) explica que una política fiscal contracíclica debe enfrentar los retos que conllevan los agudos ciclos de financiamiento externo que enfrentan los países en desarrollo. Mientras que la política monetaria debe moderar la presión hacia un manejo procíclico, generada por los ciclos de financiamiento externo, en economías que han abierto sus cuentas de capitales.

Tabla 8. Sincronización del Ciclo de Política Fiscal y el Ciclo de Negocios de Colombia

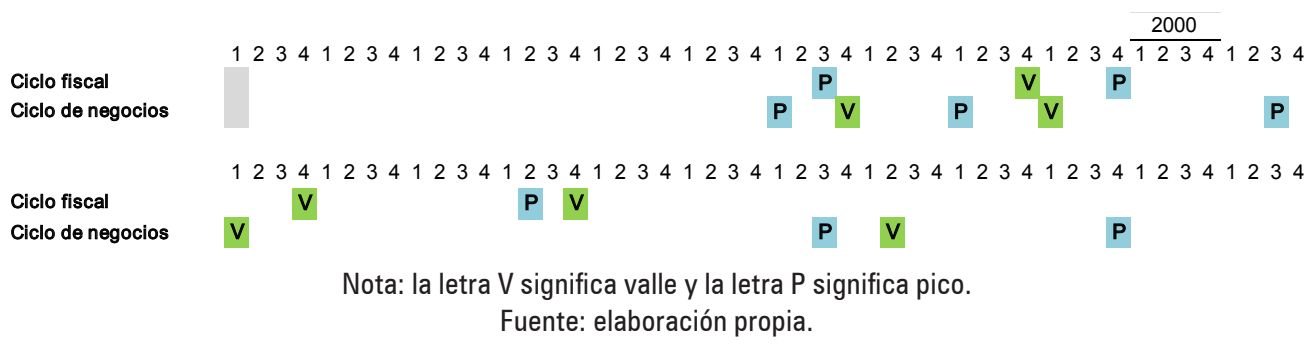

Tabla 9. Sincronización del Ciclo de Política Monetaria y el Ciclo de Negocios de Colombia 2000

123412341234123412341234123412341234123412341234

Ciclo de negocios Ciclo monetario
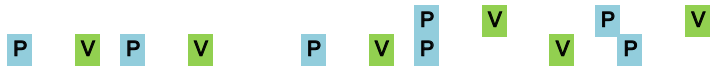

V P 


\section{CONCLUSIONES}

En este estudio se retoma la diferenciación existente entre los ciclos de negocios clásicos y los ciclos de crecimiento, y se argumenta que los ciclos clásicos ofrecen una aproximación atractiva para fechar el ciclo económico. Se construye un ciclo de negocios colombiano de referencia y se examina su sincronización con los ciclos de algunas variables relacionadas con las políticas de estabilización en el país. Se toma el consumo de gobierno trimestral desde marzo de 1990 hasta junio de 2013 como representante del ciclo de política fiscal, y la tasa de captación trimestral para el mismo periodo, como representante del ciclo de política monetaria (o alternativamente de las condiciones de liquidez de la economía).

Durante el periodo analizado se evidencian tres ciclos de negocios, dos ciclos en la política fiscal y ocho ciclos en la política monetaria. Este último presenta mayor volatilidad. La cronología propuesta para el ciclo de los negocios refleja la recesión de 1998-1999 y la del 2008.

Con ayuda del estadístico de sincronización planteado por de Harding y Pagan (2006) se concluye que el 69 porciento del tiempo el ciclo de negocios y la política fiscal se encuentran en la misma fase, es decir que la política ha sido expansiva, cuando el ciclo de negocios está en una fase de auge. Por el contrario, cuando se está en una fase recesiva, la política es contractiva. Es decir, la misma tiene un comportamiento procíclico.

De la misma forma, el estadístico de sincronización, muestra que 57 por ciento del tiempo, el ciclo de negocios y la política monetaria se encuentran en la misma fase. Lo que esto indica es que, o bien la política monetaria es acíclica o bien la misma no se transmite a la tasa de interés de mercado pasiva, ya que esta última es acíclica (con respecto al ciclo de referencia).

Se concluye por tanto que la política fiscal y monetaria no están jugando el papel contracíclico que teóricamente deberían desempeñar para estabilizar la economía. Esto se conoce en la literatura como el fenómeno "when it rains, it pours" y tiende a ser un lugar común en las economías emergentes.

Vale la pena anotar que este estudio no pretende realizar aseveraciones de naturaleza causal entre los ciclos, para lo que sería necesario imponer restricciones teóricas precisas sobre las dinámicas de las series bajo análisis, e incluirlas dentro de un sistema de ecuaciones más amplio, que además tuviese en cuenta posibles efectos rezagados de la política. Constituye por tanto, un examen de correlaciones, aun cuando modificadas, ya que se debe lidiar con la no estacionariedad de las 
series en niveles, por cual es necesario recurrir a estadísticos de concordancia. No obstante, se trata de un punto de partida para nuevos análisis, que además podrían involucrar otras variables reales en la determinación del ciclo, tales como series de empleo, distintas tasas de interés reales, variables de cuentas externas, entre otras.

Por último, este estudio busca crear interés y una base para futuras investigaciones sobre la política de estabilización y su efecto sobre el ciclo de los negocios colombianos, como un incentivo para que en Colombia se cree una institución encargada del estudio de la actividad económica como lo hace el National Bureau of Economic Research, en Estados Unidos.

\section{BIBLIOGRAFÍA}

Alfonso, Viviana; Arango, Eduardo; Arias, Fernando; Cangrejo, Guillermo y Pulido, José David (2012). Ciclos de negocios en Colombia: 1975-2011. En: Borradores de Economía, Banco de la República de Colombia, No. 651, p. 1-57.

Arango, Luis Eduardo; Arias, Fernando y Flórez (2007). Cronología de los ciclos de crecimiento recientes en Colombia. En: Borradores de Economía, Banco de la República de Colombia, No. 464 , p. 1-23.

Arango, Luis Eduardo; Arias, Fernando; Flórez, Luz Adriana y Jalil, Munir (2008). Cronología de los ciclos de negocios recientes en Colombia. En: Lecturas de Economía, Vol. 68, No. 68, p. 9-37.

Avella, Mauricio y Fergusson, Leopoldo (2004). El ciclo económico - Enfoques e ilustraciones Los ciclos económicos de Estados Unidos y Colombia. En: Borradores de Economía, Banco de la República de Colombia, No. 284, p. 1-78.

Baxter, Marianne y King, Robert (1999). Measuring business cycles: approximate band-pass filters for economic time series. En: Review of Economics and Statistics, Vol. 8, No. 4, p. 575-593.

Beveridge, Stephen y Nelson, Charles (1981). A new approach to decomposition of economic time series into permanent and transitory components with particular attention to measurement of the business cycle. En: Journal of Monetary Economics, Vol. 7, No. 2, p. 151-174.

Boschan, Charlotte y Ebanks, Walter (1978). The phase average trend: a new way of measuring economic growth. En: Proceedings of the Business and Economic Statistics Section, American Statistics Association, p. 332-335.

Bry, Gerhard y Boschan, Charlotte (1971). Cyclical analysis of time series: selected procedures and computer programs. New York, NBER, $216 \mathrm{p}$.

Burns, Arthur y Mitchell, Wesley (1946). Measuring business cycles. New York, NBER, 590 p.

Canova, Fabio (1998). Detrending and business cycle facts. En: Journal of Monetary Economics, Vol. 41, p. 475-512.

Canova, Fabio y Schlaepfer, Alain (2012). Has the euro-mediterranean partnership affected mediterranean business cycles? En: Economics Working Papers, Department of Economics and Business, Universitat Pompeu Fabra, working paper No. 1267, p. 1-40. 
Christiano, Lawrence y Fitzgerald, Terry (2003). The band pass filter. En: International Economic Review, Vol. 44, No. 2, p. 435-465.

Currie, Lauchlin (1968). Desarrollo económico acelerado: la necesidad y los medios. México, Fondo de Cultura Económica, 299 p.

Currie, Lauchlin (1993). La teoría en que se basa la estrategia del sector líder. En: Cuadernos de Economía, Vol. 13, No. 18-19, p. 225-230.

Galí, Jordi (2015). Monetary policy, inflation, and the business cycle : an introduction to the new Keynesian framework and its applications. Princeton, Princeton University Press, 296 p.

Hamilton, James (2011). Calling recessions in real time. En: International Journal of Forecasting, Vol. 27, No. 4, p. 1006-1026.

Harding, Don y Pagan, Adrian (2002). Dissecting the cycle: a methodological investigation. En: Journal of Monetary Economics, Vol. 49 No. 2, p. 365-381.

Harding, Don y Pagan, Adrian (2005). A suggested framework for classifying the modes of cycle research. En: Journal of Applied Econometrics, Vol. 20, No. 2, p. 151 159.

Harding, Don y Pagan, Adrian (2006). Synchronization of cycles. En: Journal of Econometrics, Vol. 132, No. 1, p. 59-79.

Hirschman, Albert (1983). La estrategia del desarrollo económico. En: El Trimestre Económico, Vol. 50, No.199(3), p. 1331-1424.

Kaminsky, Graciela; Reinhart, Carmen y Végh, Carlos (2004). When It rains, It pours: procyclical capital flows and macroeconomic policies. En: NBER Macroeconomics Annual 2004, Vol. 19, p. 11-82.

Kydland, Finn; Prescott, Edward y Todd, Richard (1990). Business cycle: real facts and a monetary myth. En: Federal Reserve Bank of Minneapolis Quarterly Review, Vol. 14, No. 2, p. 3-18.

Lucas Jr, Robert (1977). Understanding business cycles. En: Stabilization of the Domestic and International Economy. Amsterdam, North-Holland, p. 7-29

Melo, Alberto; French, Mercedes y Langebaek, Nils (1988). El ciclo de referencia de la economía colombiana. En: Revista Hacienda No. 12, p. 43-61.

Mintz, Ilse (1974). Dating united states growth cycles. En: Explorations in economic research NBER, Vol. 1, No. 1, p. 1-113.

Ocampo, Jose (2011). Macroeconomía para el desarrollo: políticas anticíclicas y transformación productiva. En: Revista de la CEPAL, No. 104, p. 7-35.

Posada, Carlos Esteban (1999). Los ciclos económicos colombianos en el siglo XX. En: Borradores de Economía, Banco de la República de Colombia, No. 126, p. 1-73.

Reinhart, Carmen y Reinhart, Vincent (2009). Capital flow bonanzas: an encompassing view of the past and present. En: NBER International Seminar on Macroeconomics 2008, Chicago, University of Chicago $\overline{\text { Press, p. 9-62 }}$

Schumpeter, Joseph (1935). The analysis of economic change. En: Review of Economics and Statistics, Vol. 17, No. 4, p. 2-10. 
Spiegel, Shari (2007). Políticas macroeconómicas y de crecimiento - Estrategias nacionales de desarrollo - Guías de orientación de políticas públicas. (En línea) UN DESA, Naciones Unidas, Nueva York, junio de 2007, p. 1-73 < http://esa.un.org/techcoop/documents/macro _ spanish. pdf $>$. $(13 / 11 / 2015)$.

Spiethoff, Arthur (1925). Krisen. En: Handelswörterbuch der Staatwisenschaften, Vol. VI.

Woodford, Michael (2003). Interest and prices : foundations of a theory of monetary policy. Princeton, Princeton University Press, 808 p. 